\title{
CORRIGENDUM
}

\section{THE VAPOUR PRESSURE EQUATION OF SOLUTIONS AND THE OSMOTIC PRESSURE OF RUBBER*}

\author{
BY A. R. MILLER
}

Received 23 March 1943

A number of errors have unfortunately been made in the last section of the above paper.

(1) ' $\log$ ' should be deleted from equation (44).

(2) The simplification of equation (45) which was presented neglects some important terms, with the result that the later equations give only the limiting values of osmotic pressure and vapour pressure at infinite dilution. The section following equation (45) must therefore be ignored.

Expansion of the log terms of equation (45), neglecting terms beyond the second, leads to the expression

$$
\pi=\frac{\mathbf{R} T}{\left[v_{\beta}\right]_{0}}\left[\frac{1}{n}\left(\frac{n N_{\alpha}}{N_{\beta}+n N_{\alpha}}\right)+\left\{\frac{1}{2}-\frac{1}{z}\left(1-\frac{1}{n}\right)^{2}\right\}\left(\frac{n N_{\alpha}}{N_{\beta}+n N_{\alpha}}\right)^{2}+\ldots\right] .
$$

Now $\frac{n N_{\alpha}}{N_{\beta}+n N_{\alpha}}=v_{r}$, the volume fraction of rubber in the solution. Further, if $V_{r}$ is the partial molar volume of the rubber in the solution, then $n=V_{r} /\left[v_{\beta}\right]_{0}$, and equation (1) becomes

$$
\pi \simeq \mathbf{R} T\left[\frac{v_{r}}{V_{r}}+\left(\frac{1}{2}-\frac{1}{z}\right) \frac{v_{r}^{2}}{\left[v_{\beta}\right]_{0}}\right]
$$

If $c$ is the concentration of the solution (g./c.c.) and $\rho_{r}, M_{r}$ are the density and molecular weight of the rubber, we may write (2) in the form

$$
\frac{\pi}{c} \simeq \frac{\mathbf{R} T}{M_{r}}+\left(\frac{1}{2}-\frac{1}{z}\right) \frac{\mathbf{R} T}{\rho_{r}^{2}\left[v_{\beta}\right]_{0}} c .
$$

This is in agreement with the form of equation given by Flory(1) and Huggins(2), differing only in the coefficient of the quadratic term.

To the same degree of approximation, the vapour pressure of a dilute solution is obtained by combining the above result with equation (44) of the paper, giving

$$
\frac{\Delta p_{\beta}}{\left[p_{\beta}\right]_{0}}=\frac{\left[v_{\beta}\right]_{0}}{M_{r}} c+\frac{\left(\frac{1}{2}-\frac{1}{z}\right)}{\rho_{r}^{2}} c^{2}
$$

* Proc. Cambridge Phil. Soc. 39 (1943), 54.

\section{REFERENCES}

(1) J. Chem. Phys. 9 (1941), 660; 10 (1942), 51.

(2) J. Chem. Phys. 9 (1941), 440; J. Phys. Chem. 46 (1942), 1.

\section{Gonville and Caius College}

CAmbridae 